\title{
Toll-like receptor family members in skin fibroblasts are functional and have a higher expression compared to skin keratinocytes
}

\author{
CHENG YAO ${ }^{1-3}$, JANG-HEE OH ${ }^{1-3}$, DONG HUN LEE ${ }^{1-3}$, JUNG-SOO BAE ${ }^{1-3}$, \\ CHENG LONG JIN ${ }^{1-4}$, CHI-HYUN PARK ${ }^{1-3^{*}}$ and JIN HO CHUNG ${ }^{1-3 *}$ \\ ${ }^{1}$ Department of Dermatology, Seoul National University College of Medicine; \\ ${ }^{2}$ Institute of Human-Environment Interface Biology, Medical Research Center, Seoul National University; \\ ${ }^{3}$ Laboratory of Cutaneous Aging Research, Biomedical Research Institute, Seoul National University Hospital, Seoul 110-744, \\ Republic of Korea; ${ }^{4}$ Department of Dermatology, Yanbian University Hospital, Yanji, Jilin 133002, P.R. China
}

Received July 7, 2014; Accepted March 6, 2015

DOI: 10.3892/ijmm.2015.2146

\begin{abstract}
Toll-like receptors (TLRs) are known to recognize not only pathogen-associated molecular patterns but also danger-associated molecular patterns. Recent studies have characterized the expression levels and functions of TLRs in human epidermal cells. However, the characteristics of TLR family members in human dermal fibroblasts have not been thoroughly studied. Therefore, the present study systematically investigated the expression levels of TLRs and their functional responses to each ligand in skin fibroblasts. All 10 TLRs are expressed in skin fibroblasts. Stimulation of skin fibroblasts with each TLR ligand resulted in an increase of the interleukin-6 (IL-6), IL-8 and matrix metalloproteinase-1 proteins, indicating that $\geq 9$ TLRs in skin fibroblasts are functionally active. Furthermore, stimulating skin fibroblasts with TLR1/2, 3 and 4 ligands induced the phosphorylation of inhibitor of nuclear factor $\kappa \mathrm{B} \alpha$ and the active phosphorylation of extracellular-signal regulated kinase $1 / 2$. The expression level of each TLR was much higher in fibroblasts compared to keratinocytes. In particular, the fold-increase in $I L-6$ and $I L-8$ mRNA levels upon exposure to a TLR1/2 ligand was much higher in fibroblasts compared to keratinocytes, which appears to reflect the difference in expression levels of TLR1 and 2 between fibroblasts and keratinocytes. Taken together, these results show that all 10 TLRs are constitutively expressed and functional (except TLR10) in skin fibroblasts and suggest that
\end{abstract}

Correspondence to: Professor Jin Ho Chung, Department of Dermatology, Seoul National University College of Medicine, 28 Yongon-dong, Chongno-gu, Seoul 110-744, Republic of Korea E-mail: jhchung@snu.ac.kr

*Joint senior authorship

Key words: fibroblasts, keratinocytes, Toll-like receptors, interleukin-6, interleukin-8, matrix metalloproteinase-1
TLRs in skin fibroblasts may play an important role in the detection of and response to different classes of pathogens and danger signals.

\section{Introduction}

Toll-like receptors (TLRs) are a group of receptors that recognize conserved pathogen-associated molecular patterns (PAMPs) $(1,2)$, playing a key role in the host defense against infection. Thus far, 10 members of TLRs with distinct PAMP ligands have been identified in humans (3). Triacyl lipopeptide and diacyl lipopeptide are recognized by TLR1/2 and TLR2/6, respectively (4-6). TLR3 recognizes double-stranded RNA (7). The receptor for lipopolysaccharide (LPS) is TLR4 (8). TLR5 recognizes bacterial flagellin (9). Imidazoquinolines and single-stranded RNA (ssRNA) are recognized by TLR7 and TLR8 (10). TLR9 recognizes bacterial and viral CpG DNA motifs (11). In addition to sensing exogenous ligands from microbial components, TLRs can also recognize endogenous ligands, known as danger-associated molecular patterns (12). For example, it is well-known that heat-shock proteins (HSPs) can be locally released upon injury and subsequently activate TLR2 or 4 (13). Certain self non-coding RNAs damaged by ultraviolet (UV) radiation and released from necrotic cells can be recognized by TLR3 to induce inflammatory cytokines (14).

The TLR-mediated signaling pathways can be divided into two major pathways: Myeloid differentiation primary response protein 88 (MyD88)- and TIR-domain-containing adapter-inducing interferon- $\beta$ (TRIF)-dependent pathways. MyD88 is an adapter protein used by almost all 10 TLRs (except TLR3) to initiate TLR signaling (3). However, the signal transduction pathway of TLR3 requires the other adaptor protein, TRIF (3). TLR4 can utilize MyD88 and TRIF as signaling adaptors $(3,15)$. Following recognition of different ligands, TLRs trigger signaling pathways and activate nuclear factor (NF)- $\mathrm{B}$ and activator protein-1 (AP-1), which results in the induction of inflammatory cytokines and chemokines $(2,3)$.

In skin, TLRs are expressed not only in professional immune cells (such as Langerhans cells) but also in non-immune cells 
(including keratinocytes and melanocytes) (16-18). Several studies have shown that skin keratinocytes and melanocytes exhibit TLRs and respond to corresponding PAMPs by producing pro-inflammatory cytokines $(17,18)$. Although recent studies have reported that TLRs are expressed in skin fibroblasts $(19,20)$, the expression levels and functional responses of TLRs in skin fibroblasts have not been thoroughly studied.

In the present study, the expression levels of all the TLR family members (TLR1-10) were analyzed in skin fibroblasts. Subsequently, using a specific ligand for each TLR, the functional activation of each TLR was observed in fibroblasts. The signaling pathways induced by TLR $1 / 2,3$ and 4 were also examined. Furthermore, the expression levels of TLRs were compared in skin fibroblasts with those in skin keratinocytes. Finally, the mRNA levels of interleukin-6 (IL-6) and $I L-8$ increased upon exposure to a TLR1/2 ligand when compared between fibroblasts and keratinocytes.

\section{Materials and methods}

Reagents. All the TLR ligands were purchased from InvivoGen, Inc. (San Diego, CA, USA). For western blot analysis, antibodies to phospho-inhibitor of nuclear factor $\kappa \mathrm{B} \alpha(\mathrm{I} \kappa \mathrm{B} \alpha)$ and phosphoand total-extracellular-signal regulated kinase (ERK) $1 / 2$ were purchased from Cell Signaling Technology, Inc. (Beverly, MA, USA). The antibody to matrix metalloproteinase-1 (MMP-1) was manufactured by Lab Frontier Co., Ltd. (Seoul, Korea). Antibodies to TLR2, 3, 4 and $\beta$-actin were purchased from Santa Cruz Biotechnology, Inc. (Dallas, TX, USA).

Cell culture. Human foreskin fibroblasts and keratinocytes were established from biopsies obtained from four different healthy donors. Skin fibroblasts were cultured in Dulbecco's modified Eagle's media (DMEM) supplemented with glutamine $(2 \mathrm{mM})$, penicillin $(400 \mathrm{U} / \mathrm{ml})$, streptomycin $(50 \mathrm{mg} / \mathrm{ml})$ and $10 \%$ fetal bovine serum (FBS) in a humidified $5 \% \mathrm{CO}_{2}$ atmosphere at $37^{\circ} \mathrm{C}$. Skin fibroblasts were used for the experiments at passages 3-6. For treatment, skin fibroblasts were serum-starved for $48 \mathrm{~h}$ in DMEM containing 0.1\% FBS.

Skin keratinocytes were cultured in keratinocyte growth medium (Clonetics, Inc., San Diego, CA, USA) composed of MCDB 153 medium supplemented with epidermal growth factor $(10 \mathrm{ng} / \mathrm{ml})$, bovine pituitary extract $(70 \mu \mathrm{g} / \mathrm{ml})$, hydrocortisone $(0.5 \mu \mathrm{g} / \mathrm{ml})$, penicillin $(100 \mu \mathrm{g} / \mathrm{ml})$, streptomycin $(100 \mu \mathrm{g} / \mathrm{ml})$ and fungizone $(0.25 \mu \mathrm{g} / \mathrm{ml})$. Skin keratinocytes were used at passage 3-4 in the experiments. For treatment, skin keratinocytes were maintained in keratinocyte basal medium (MCDB 153 medium only) for $48 \mathrm{~h}$.

Ligand treatment. To induce IL-6, IL-8 and MMP-1 production, skin fibroblasts were plated at the concentration of $0.5 \times 10^{5}$ cells $/ 500 \mu \mathrm{l}$ in 24 -well plates for $48 \mathrm{~h}$ in the presence of different concentrations of Pam3CSK4 (TLR1/2 ligand; $0.01,0.1$ and $1 \mu \mathrm{g} / \mathrm{ml}$ ), poly (I:C) (TLR3 ligand; $0.1,1$ and $10 \mu \mathrm{g} / \mathrm{ml}$ ), LPS (TLR4 ligand; 0.001, 0.01 and $0.1 \mu \mathrm{g} / \mathrm{ml}$ ), flagellin (TLR5 ligand; $0.05,0.5$ and $5 \mu \mathrm{g} / \mathrm{ml}$ ), FSL-1 (TLR2/6 ligand; $0.001,0.01$ and $0.1 \mu \mathrm{g} / \mathrm{ml}$ ), imiquimod (TLR7 ligand; $0.1,1$ and $10 \mu \mathrm{g} / \mathrm{ml}$ ), ssRNA40 (TLR8 ligand; 0.1, 1 and $10 \mu \mathrm{g} / \mathrm{ml}$ ) and ODN2006 (TLR9 ligand, 0.05, 0.5 and $5 \mu \mathrm{M}$ ).
The concentrations of each TLR ligand were screened and determined using the information in previously published studies $(17,18)$.

RNA isolation, and semi-quantitative and reverse transcription-quantitative polymerase chain reaction ( $R T-q P C R)$. Total RNA was isolated using TRIzol (Invitrogen, Carlsbad, CA, USA). Total RNA ( $1 \mu \mathrm{g}$ ) was used to generate cDNA using a First Strand cDNA Synthesis kit (MBI Fermentas, Vilnius, Lithuania) according to the manufacturer's instructions.

Semi-quantitative RT-PCR. Semi-quantitative RT-PCR was performed to detect TLRI-10 and $36 B 4 \mathrm{mRNA}$ using PCR Premix (Promega, Madison, WI, USA). PCR conditions were as follows: $95^{\circ} \mathrm{C}$ for $2 \mathrm{~min}, 35$ cycles of $94^{\circ} \mathrm{C}$ for $30 \mathrm{sec}, 60^{\circ} \mathrm{C}$ for $30 \mathrm{sec}$ and $72^{\circ} \mathrm{C}$ for $1 \mathrm{~min}$. The PCR products were visualized on $2 \%$ agarose gels using ethidium bromide staining. The primer sequences for semi-quantitative RT-PCR are shown in Table I.

$R T-q P C R$. RT-qPCR was performed to measure the relative levels of TLR1-10 mRNA using an ABI Prism 7500 (Applied Biosystems, Darmstadt, Germany). Input cDNA was normalized according to $36 B 4$ or actin as internal control genes. The primer sequences for RT-qPCR were the same as for semi-quantitative RT-PCR.

Enzyme-linked immunosorbent assay (ELISA). After stimulation for $48 \mathrm{~h}$ with TLR ligands, cell culture supernatants were harvested and examined by ELISA for IL-8 and IL-6, following the manufacturer's instructions (Endogen, Inc., Woburn, MA, USA).

Western blotting. For the MMP-1 measurement, an equal volume of cell culture supernatants was prepared for western blot analysis. $\beta$-actin was detected from an equal volume of cell lysate as a loading control for MMP-1. For the detection of protein in cell lysates, cells were washed twice with ice-cold phosphate-buffered saline to remove all the serum proteins and were subsequently lysed in cell lysis buffer. Separated proteins were transferred to polyvinylidene fluoride membranes, which were subsequently blocked with Tris-buffered saline containing $0.1 \%$ Tween-20 and 5\% dried skimmed milk. Membranes were incubated with the appropriate primary antibody and horseradish peroxidase-conjugated secondary antibody. Antibody-bound proteins were visualized by enhanced chemiluminescence (GE Healthcare, Buckinghamshire, UK).

Statistical analysis. Statistical analyses were performed using the Student's t-test. $\mathrm{P}<0.05$ was considered to indicate a statistically significant difference. Results are presented as mean values \pm standard error of the mean.

\section{Results}

All 10 TLR family members are expressed in human skin fibroblasts. In order to gain insight into the functions of TLRs in skin fibroblasts, the expression levels of TLR family members were assessed by RT-qPCR. Consistent with the previously published studies $(17,19,20)$, all the 10 TLR family members were constitutively expressed in skin fibroblasts (Fig. 1). 
Table I. Primer sequences.

\begin{tabular}{lll}
\hline Gene & \multicolumn{1}{c}{ Forward primer } & \multicolumn{1}{c}{ Reverse primer } \\
\hline$T L R 1$ & CTATACACCAAGTTGTCAGC & GTCTCCAACTCAGTAAGGTG \\
$T L R 2$ & GCCAAAGTCTTGATTGATTG & TTGAAGTTCTCCAGCTCCTG \\
$T L R 3$ & GATCTGTCTCATAATGGCTTG & GACAGATTCCGAATGCTTGTG \\
$T L R 4$ & TGGATACGTTTCCTTATAAG & GAAATGGAGGCACCCCTTC \\
$T L R 5$ & ATTGCCAATATCCAGGATGC & CACCACCATGATGAGAGCAC \\
$T L R 7$ & TTGGGCTAACATTAGAGCCG & AGCTCAGTTCCCCAGATGAA \\
$T L R 8$ & AATGTCACAGCCGTCCCTAC & GCGCATCAAAAGCATTTACA \\
$T L R 9$ & TCAACAAATCCGCACTTGAA & CAGGACTGGCACAAATGACA \\
$T L R 10$ & TACGATGCCTTCGTGGTCTT & CTCAAAGAGGGTTTGCCAG \\
$\beta-$-actin & TCTCCCTGGATGCAGTCATT & AACTTCCTGGCAGCTCTGAA \\
$I L-6$ & TGGGCTCCAAGCAGATGC & GGCTTCGCTGGCTCCCAC \\
$I L-8$ & AGAGATGGCCACGGCTGCTT & ATTTGCGGTGGACGATGGAG \\
\hline
\end{tabular}

TLR, Toll-like receptor; IL, interleukin.

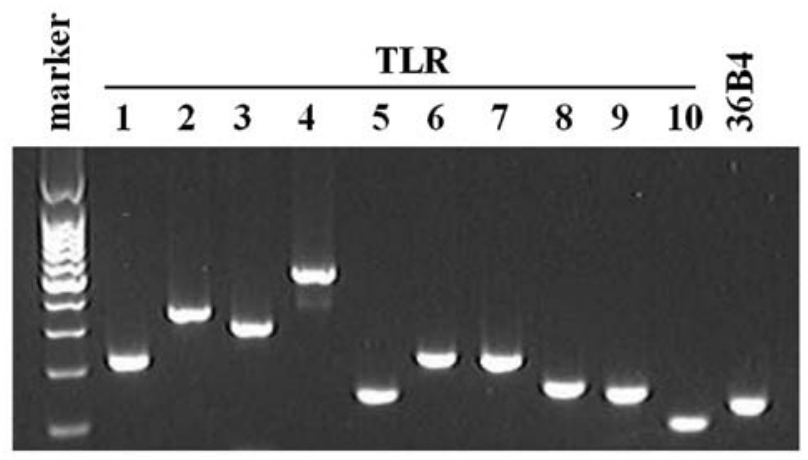

Figure 1. All 10 TLR family members are expressed in human skin fibroblasts. Total RNA was prepared from cultured skin fibroblasts and subjected to RT-PCR using TLR1-10 specific primers. The PCR products were visualized on $2 \%$ agarose gels using ethidium bromide staining. The data shown are representative of three independent experiments. TLR, Toll-like receptors; RT-PCR, reverse transcription-polymerase chain reaction.

Activation of TLRs induces expression of $I L-6, I L-8$ and MMP-1 in human skin fibroblasts. Activation of TLRs in skin keratinocytes and melanocytes are known to produce various cytokines and chemokines $(17,18,21)$. As all 10 TLRs are expressed in skin fibroblasts, whether these TLRs are functional or not was questioned. Therefore, skin fibroblasts were stimulated with each available TLR ligand (TLR10 ligand was unavailable for purchasing) for $48 \mathrm{~h}$ and the protein expression of IL- 6 and IL-8 induced by each TLR ligand was analyzed. The concentrations of each TLR ligand were screened and determined using the information in previously published studies $(17,18)$. IL-6 and IL-8 were selected as representatives of cytokines and chemokines, respectively. All the TLR ligands induced the production of IL-6 (Fig. 2A) and IL-8 (Fig. 2B) in a dose-dependent manner, except IL-8 and IL-6 in the activation of TLR8 and 9, respectively. Activation of TLR8 and 9 induced IL- 6 and IL- 8 compared to the activation of the other TLRs.
The expression of members of the MMP family is important during inflammation, cell migration and during the remodeling of damaged tissues $(22,23)$. In addition to IL-6 and IL-8, whether the activation of TLRs in skin fibroblasts induced MMPs, such as MMP-1, was investigated. All the TLR ligands induced the production of MMP-1 in a dose-dependent manner (Fig. 3). Activation of TLR8 and 9 induced MMP-1 compared to the activation of the other TLRs.

Taken together, these results suggest that TLR1-9 in human skin fibroblasts are functional and that activation of these TLRs can induce the expression of IL-6, IL-8 and MMP-1.

Triggering TLRI/2, 3 and 4 by each ligand induces phosphorylation of $I \kappa B \alpha$ and activation of ERK in human skin fibroblasts. Activation of $\mathrm{NF}-\kappa \mathrm{B}$ and mitogen-activated protein (MAP) kinases has been shown to play a central role in TLR-mediated cellular activation and gene expression in a variety of cell types $(17,18,21)$. Therefore, whether triggering TLRs with each ligand can induce the activation of $N F-\kappa B$ and MAP kinase signaling pathways in skin fibroblasts was tested. Skin fibroblasts were stimulated with TLR1/2, 3 and 4 ligands and harvested at $3 \mathrm{~h}$ after treatment. The samples were assessed by western blot analysis for the phosphorylation of $\mathrm{I} \kappa \mathrm{B} \alpha$, which is frequently used as one of the markers for activation of the NF- $\kappa \mathrm{B}$ pathway, and active phosphorylation of ERK, which is representative of MAP kinases. Treatment of TLR1/2, 3 and 4 ligands induced phosphorylation of $\mathrm{I} \kappa \mathrm{B} \alpha$ and active phosphorylation of ERK (Fig. 4).

In conclusion, the data suggest that activation of TLR can induce activation of the NF- $\mathrm{B}$ and ERK pathways in skin fibroblasts.

Expression levels of TLR family members are higher in skin fibroblasts compared to skin keratinocytes. Skin keratinocytes are the major cells expressed in the epidermis, which is the 
A

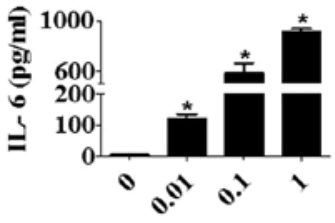

Pam3CSK4

$(\mu \mathrm{g} / \mathrm{ml})$

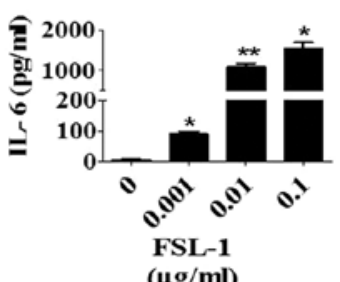

B

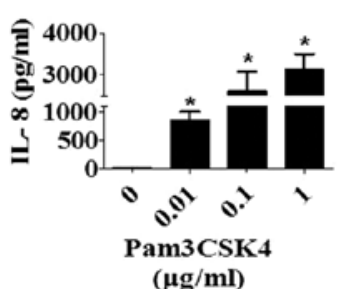

$(\mu \mathrm{g} / \mathrm{mI})$

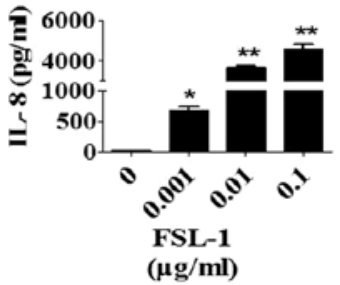

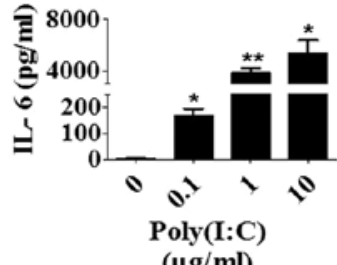

$(\mu \mathrm{g} / \mathrm{ml})$

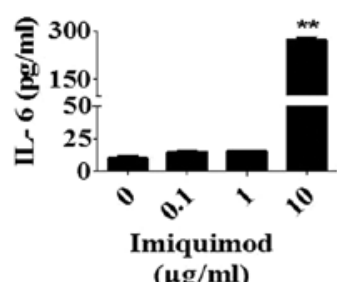

$(\mu \mathrm{g} / \mathbf{m l})$
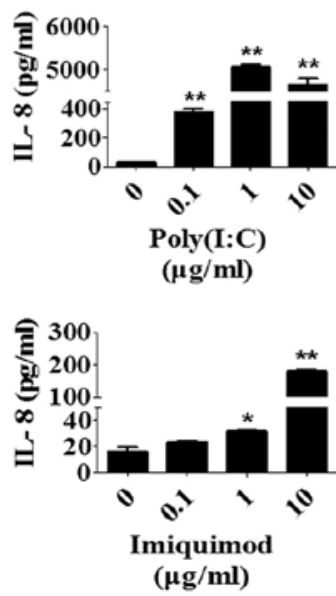
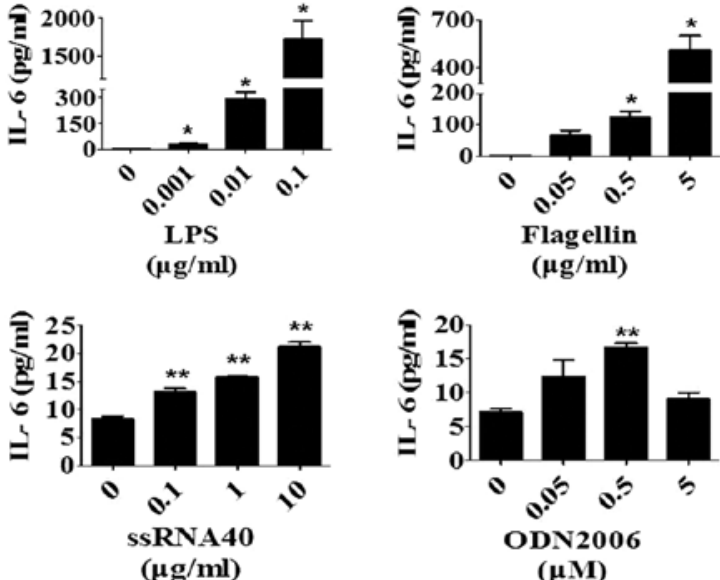

$(\mu \mathrm{M})$
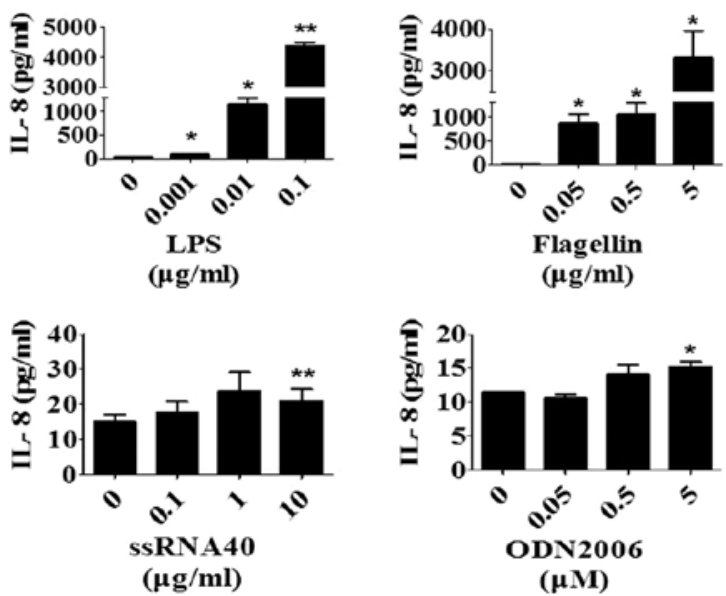

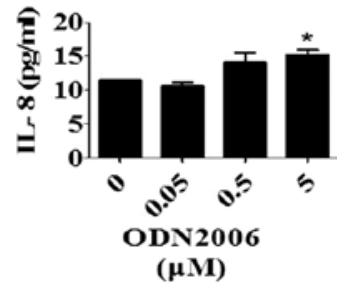

Figure 2. Activation of TLRs induces the expression of IL-6 and IL-8 in human skin fibroblasts. After culture in basal medium for 48 h, skin fibroblasts were incubated with different concentrations of Pam3CSK4 (TLR1/2 ligand), poly (I:C) (TLR3 ligand), LPS (TLR4 ligand), flagellin (TLR5 ligand), FSL-1 (TLR2/6 ligand), imiquimod (TLR7 ligand), ssRNA40 (TLR8 ligand) and ODN2006 (TLR9 ligand). At 48 h after treatment, the concentrations of (A) IL-6 and (B) IL-8 in the cell culture medium were measured with specific ELISA. The results expressed as mean \pm standard error of the mean of triplicate cultures are from one experiment representative of three with different donors. ${ }^{*} \mathrm{P}<0.05,{ }^{* *} \mathrm{P}<0.01$ vs. corresponding control. TLR, Toll-like receptor; IL, interleukin; LPS, lipopolysaccharide; ssRNA, single-stranded RNA; ELISA, enzyme-linked immunosorbent assay.

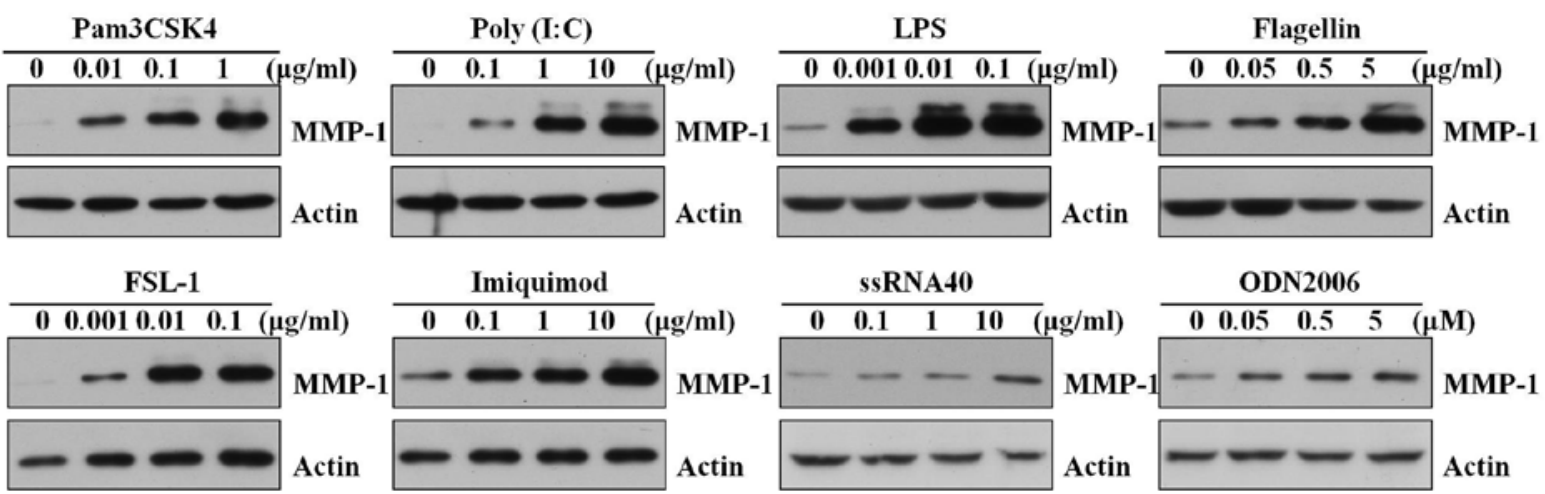

Figure 3. Activation of TLRs induces expression of MMP-1 protein in human skin fibroblasts. At 48 h after incubation with different TLR ligands, protein levels of MMP-1 in the cell culture medium and actin in the cell lysates were analyzed with western blotting. The data shown are representative of three experiments. TLR, Toll-like receptor; MMP-1, matrix metalloproteinase-1.

first defense line of the skin against pathogens. The expression levels of TLRs were well-studied in skin keratinocytes in several research studies $(17,24)$. To improve the understanding of the TLR expression levels in skin fibroblasts, the expression levels of TLRs in skin keratinocytes were compared with fibroblasts. 


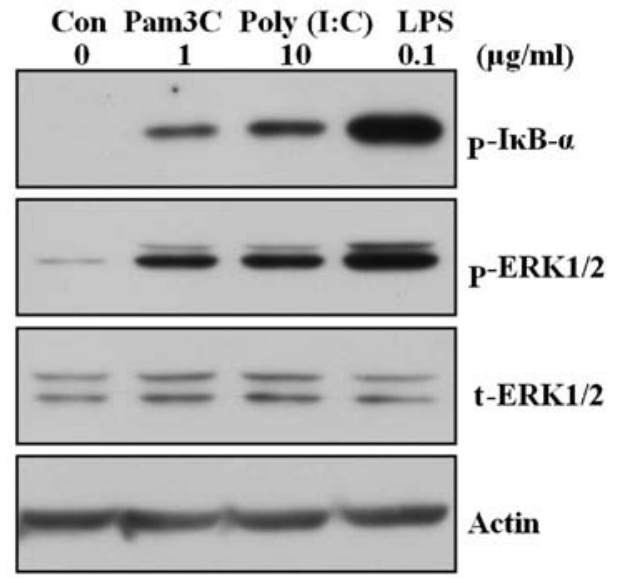

Figure 4. Triggering TLR1/2, 3 and 4 by each ligand induces phosphorylation of I $\mathrm{B} \mathrm{B} \alpha$ and activation of ERK in human skin fibroblasts. Cultured skin fibroblasts were stimulated with Pam3CSK4 $(1 \mu \mathrm{g} / \mathrm{ml})$, poly (I:C) $(10 \mu \mathrm{g} / \mathrm{ml})$ and LPS $(0.1 \mu \mathrm{g} / \mathrm{ml})$ for $3 \mathrm{~h}$. Cell lysates were fractionated by SDS-PAGE and the protein levels of $\mathrm{p}$-I $\mathrm{KB} \alpha$ and $\mathrm{p}$-ERK1/2 were analyzed by western blotting. A single representative experiment is shown from three different experiments. TLR, Toll-like receptor; I $\mathrm{B} \alpha$; inhibitor of nuclear factor $\kappa \mathrm{B} \alpha$; ERK, extracellular-signal regulated kinase; LPS, lipopolysaccharide.

To eliminate the possibility that the expression level of TLRs could be affected by different cell culture conditions, growth culture (Fig. 5A) and basal culture conditions (Fig. 5B) were used. Consistent with the previously published studies (17), all the TLRs were constitutively expressed in skin keratinocytes, except TLR7 and 8 (Fig. 5A and B). Regardless of culture conditions, each TLR was expressed much higher in skin fibroblasts compared to skin keratinocytes (Fig. 5A and B). Furthermore, these results were not dependent on different endogenous controls, as the same results were almost obtained when $\beta$-actin was used as an endogenous control (data not shown). Subsequently, the protein expression levels of certain TLRs between fibroblasts and keratinocytes were confirmed. The protein expression levels of TLR2, 3 and 4 are much higher in skin fibroblasts compared to keratinocytes (Fig. 5C).

Taken together, the data indicate that the expression level of each TLR was higher in skin fibroblasts compared to skin keratinocytes.

Fold increases in IL-6 and IL-8 mRNA expression level upon exposure to a TLR1/2 ligand are much higher in skin fibroblasts compared to skin keratinocytes. To confirm the competence of TLR signaling in skin fibroblasts, the activation of TLR1/2 signaling pathways in skin fibroblasts was compared with those in skin keratinocytes. Fibroblasts and keratinocytes were treated with Pam3CSK4 and the cells were harvested at 10 and $24 \mathrm{~h}$ after treatment. Subsequently, the mRNA levels of $I L-6$ and $I L-8$ were analyzed by RT-qPCR, and the fold-increase was calculated in fibroblasts and keratinocytes. Pam3CSK4 treatment increased mRNA levels of $I L-6$ (Fig. 6A) and $I L-8$ (Fig. 6B) in fibroblasts and keratinocytes. However, at the two time points and concentrations, the fold-increase in $I L-6$ and $I L-8$ mRNA expression levels induced by Pam3CSK4 treatment was much higher in fibroblasts compared to keratinocytes.

Therefore, these results indicate that TLR signaling pathways in skin fibroblasts are functional and extremely active,
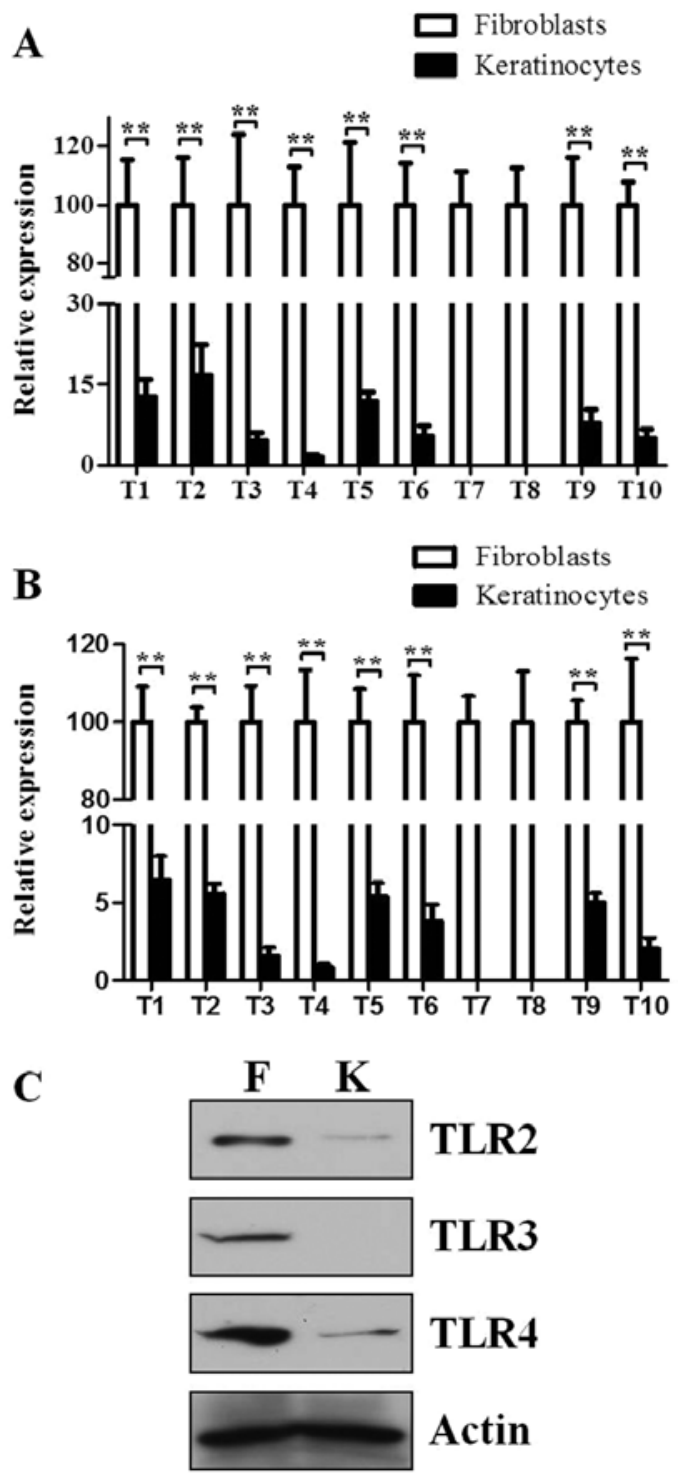

Figure 5. Expression levels of the TLR family members are higher in skin fibroblasts compared to skin keratinocytes. RNA was extracted from skin fibroblasts and keratinocytes obtained from four different donors. An equal amount of cDNA was used to assess mRNA expression of TLRs by RT-qPCR. $36 B 4$ was used as an endogenous control. (A) Cells were cultured in growth medium (DMEM containing 10\% FBS for fibroblasts and keratinocyte growth medium for keratinocytes, respectively). (B) Cells were first cultured in growth medium for $24 \mathrm{~h}$ and were maintained in basal medium (DMEM containing $0.1 \%$ FBS for fibroblasts and keratinocyte basal medium for keratinocytes, respectively) for $48 \mathrm{~h}$. Data are presented as mean \pm standard error of the mean $\left(\mathrm{n}=4 ;{ }^{* *} \mathrm{P}<0.01\right)$. (C) Cells were cultured in growth medium for $24 \mathrm{~h}$ and were maintained in basal medium, after which lysates were prepared and the protein levels of TLR2, 3 and 4 were analyzed by western blotting. A single representative experiment is shown from three different experiments. TLR, Toll-like receptor; RT-qPCR, reverse transcription-quantitative polymerase chain reaction; DMEM, Dulbecco's modified Eagle's media; FBS, fetal bovine serum; F, fibroblasts; K, keratinocytes.

and suggest that TLRs in skin fibroblasts may provide strong responses to pathogens and/or danger signals.

\section{Discussion}

In the present study, all 10 TLR family members were constitutively expressed in skin fibroblasts (Fig. 1), which is in agreement with the previously published results $(19,20)$. To 
A

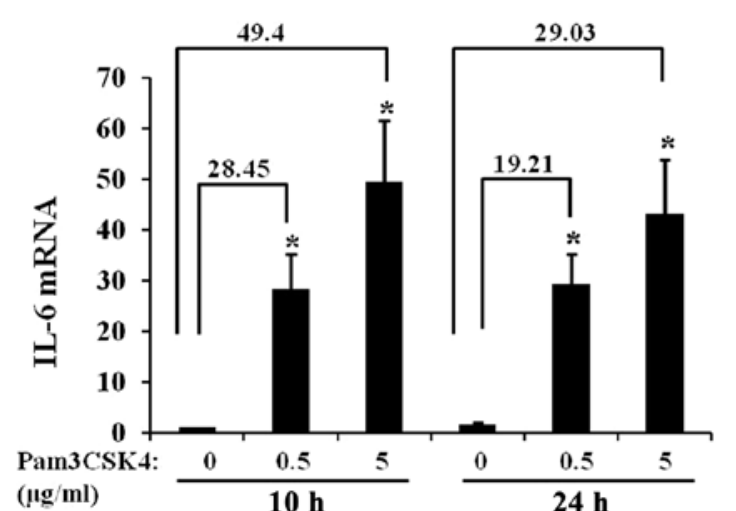

B

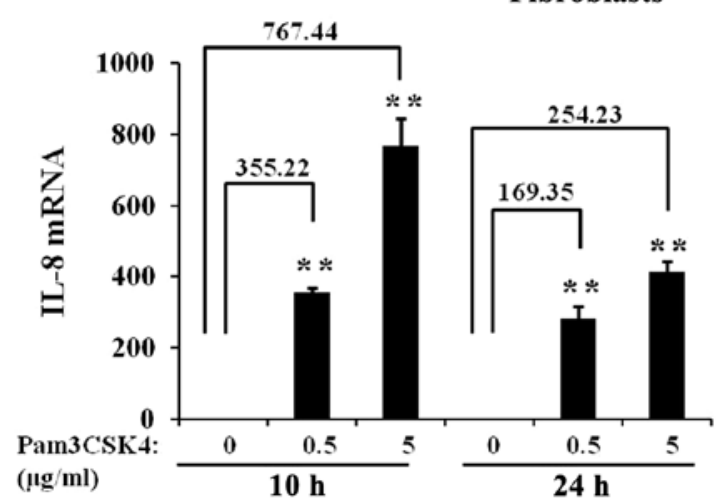

Fibroblasts

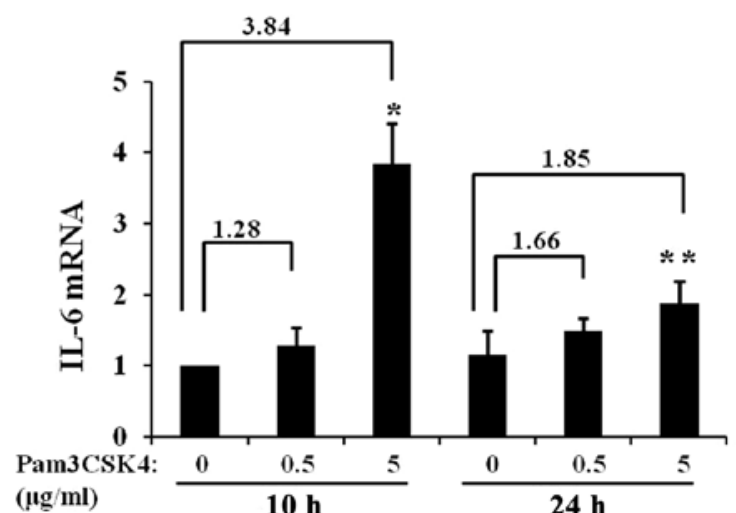

Keratinocytes

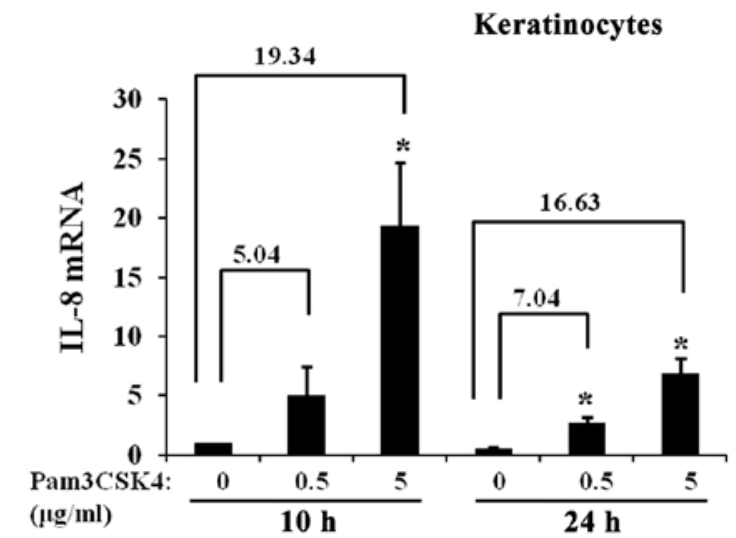

Figure 6. Fold-increase in $I L-6$ and $I L-8$ mRNA expression level upon exposure to a TLR1/2 ligand is much higher in skin fibroblasts compared to skin keratinocytes. Fibroblasts and keratinocytes were first cultured in growth medium and subsequently cultured in basal medium for $48 \mathrm{~h}$. Cells were stimulated with TLR1/2 ligand Pam3CSK4 $(0.5$ and $5 \mu \mathrm{g} / \mathrm{ml})$ for 10 and $24 \mathrm{~h}$. Following this, RNA was extracted and an equal amount of cDNA was used to detect the $I L-6$ and $I L-8$ mRNA level by RT-qPCR. Experiments were performed using fibroblasts and keratinocytes obtained from four different donors. (A) $I L-6$ and (B) $I L-8$ mRNA levels in fibroblasts and keratinocytes. Data are presented as mean \pm standard error of the mean. ${ }^{*} \mathrm{P}<0.05$, ${ }^{* *} \mathrm{P}<0.01$ vs. corresponding control. Values of the fold-increase are also shown in the graph. IL, interleukin; TLR, Toll-like receptor; RT-qPCR, reverse transcription-quantitative polymerase chain reaction.

understand whether the TLRs in skin fibroblasts are functional, skin fibroblasts were treated with each TLR ligand. Treatment with TLR1/2, 3, 4, 5, 2/6, 7, 8 and 9 ligands induced protein expression levels of IL-6, IL-8 and MMP-1, which are representatives of cytokines, chemokines and MMPs, respectively (Figs. 2 and 3). Since the effects of TLR1-9 activation by each ligand on the protein expression levels of IL-6, IL-8 and MMP-1 were not systemically examined in skin fibroblasts previously, the present data may extend the knowledge to understand expression and activation of TLRs in skin fibroblasts.

UV can directly activate growth factor or cytokine receptors on the irradiated skin cells, leading to AP-1 activation and MMP-1 upregulation, which degrades extracellular matrix proteins, including collagen (25). Decreased collagen in skin is responsible for the characteristic aged appearance of photo-damaged skin $(25,26)$. UV irradiation on keratinocytes can also induce the expression of HSP60 (27) and damage non-coding RNA (14). HSP60 is recognized in murine and human cells via TLR2 and 4 (28). UV-damaged RNA can be recognized by TLR3 in healthy keratinocytes (14). Taken together with the present finding that treatment of TLR1/2, 3 and 4 ligands can strongly induce MMP-1 expression in skin fibroblasts (Fig. 3), UV can possibly indirectly increase MMP-1 expression in skin fibroblasts through activation of
TLR pathways by an increased in the generation of endogenous TLR ligands, which may eventually contribute to photoaging.

Activation of NF- $\kappa \mathrm{B}$ and MAP kinases plays a central role in TLR-mediated cellular activation and gene expression in a variety of cell types $(17,18,21)$. NF- $\mathrm{B}$ activation is essential for the upregulation of several cytokines and chemokines, including IL-6 and IL-8 $(29,30)$. Activation of MAP kinases (ERK1/2, c-Jun N-terminal kinase and p38) is known to increase the expression of MMPs in skin cells (25). Therefore, whether triggering TLRs can give rise to activation of $N F-\kappa B$ and MAP kinases in skin fibroblasts was investigated. TLR signaling pathways can be divided into MyD88 and TRIF pathways $(3,15)$. TLR2, 3 and 4 are known to initiate signaling by MyD88, TRIF or the two pathways, respectively $(3,15)$. Thus, the ligands of TLR1/2, 3 and 4 were used to study NF- $\kappa B$ and MAP kinases signaling in fibroblasts. Pam3CSK4, poly (I:C) and LPS induced the phosphorylation of $\mathrm{I} \kappa \mathrm{B} \alpha$ (Fig. 4). Phosphorylation of $\mathrm{I} \kappa \mathrm{B} \alpha$ is known to induce the degradation of $\mathrm{I} \kappa \mathrm{B} \alpha$ and the translocation of $\mathrm{NF}-\kappa \mathrm{B}$ into the nucleus, and subsequently leads to transcription of $\mathrm{NF}-\kappa \mathrm{B}$-sensitive genes, such as $I L-6$ and $I L-8$. The present finding also supports the notion that $\mathrm{I} \kappa \mathrm{B} \alpha$ is an important cellular factor involved in the regulation of the host innate antimicrobial response (31). In addition, ERK1/2 was phosphorylated at $3 \mathrm{~h}$ after Pam3CSK4, 
poly (I:C) and LPS treatment (Fig. 4). The data suggest that MAP kinases can be activated by TLR ligands, and that the activated MAP kinases may contribute to the upregulation of MMP-1 expression by TLR ligands in skin fibroblasts.

The epidermis is generally considered as the first defense line of skin against pathogens and skin keratinocytes are major cells in the epidermis. To this point, the expression level of certain (if not all) TLR family members may be higher in skin keratinocytes compared to skin fibroblasts. Thus, the expression levels of TLRs in skin keratinocytes with fibroblasts were compared. Of note, the relative expression levels of each TLR family member were much higher in fibroblasts compared to keratinocytes, regardless of cell culture conditions. The protein expression levels of TLR2, 3 and 4 were also checked and compared between fibroblasts and keratinocytes using each specific antibody. Consistent with the mRNA levels, protein expression levels of TLR2, 3 and 4 are higher in skin fibroblasts compared to keratinocytes (Fig. 5). These results were noteworthy, considering that the epidermis is the first defense line of skin against pathogens and that skin keratinocytes are major cells in the epidermis. However, numerous studies also show that in addition to exogenous ligands derived from pathogens, various endogenous ligands can activate TLRs and initiate responses to danger signals (13-14,32).

In addition, a recent study has shown that certain TLR4 endogenous ligands, such as tenascin $\mathrm{C}$ and hyaluronic acid, are elevated in scleroderma skin lesions, and has suggested that TLR4 signaling activated by endogenous ligands in skin fibroblasts may be involved in pathogenesis of scleroderma (33). Thus, high expression levels of TLRs found in skin fibroblasts may play important roles when they are activated by exogenous and/or endogenous ligands. In addition to the cell culture system, it may also be necessary to compare the expression levels of TLRs in fibroblasts with those in keratinocytes in skin tissue. Since differentiated skin keratinocytes may have different expression levels of TLRs in skin tissue, the expression patterns of TLRs in skin tissue will be investigated in our future study. Taken together, these results show that TLRs are highly expressed in skin fibroblasts in vitro and indicate that TLRs in skin fibroblasts may play an important role on numerous physiological or pathological conditions.

Finally, to check the competence of TLR signaling pathways in skin fibroblasts, the activities of TLR signaling pathways were compared in fibroblasts with those in keratinocytes. Since the TLR1/2 signaling pathway has been well-studied in skin keratinocytes in several research studies $(34,35)$, the activities of TLR $1 / 2$ signaling pathways in fibroblasts were compared with those in keratinocytes. Fibroblasts and keratinocytes were treated with TLR1/2 ligand Pam3CSK4 for 10 and $24 \mathrm{~h}$, and the expression level of IL- 6 and IL-8 were analyzed. Since fibroblasts and keratinocytes are different types of cells, and IL- 6 and IL-8 are secreted cytokines, it may be difficult to normalize and compare the expression levels of these proteins in cell culture media between fibroblasts and keratinocytes. Therefore, the mRNA levels of $I L-6$ and $I L-8$ were analyzed by RT-qPCR, in which expression levels can be easily normalized by the same housekeeping gene. As shown in Fig. 6, Pam3CSK4 treatment increased the mRNA levels of $I L-6$ and $I L-8$ in fibroblasts and keratinocytes and the Pam3CSK4-induced expression folds of $I L-6$ and $I L-8$ were much higher in fibroblasts compared to keratinocytes at every time point and concentration. For example, after treatment with $0.5 \mu \mathrm{g} / \mathrm{ml}$ of Pam3CSK4 for $10 \mathrm{~h}$, the $I L-6$ mRNA level was increased by $\sim 28.45$-fold in skin fibroblasts but only $\sim 1.28$-fold in skin keratinocytes, as compared with their non-treated controls. These data may result, at least partially, from the fact that expression levels of TLR1 and 2 are higher in fibroblasts compared to keratinocytes (Fig. 5). Thus, the data indicate that activation of the TLR signaling pathway induced by Pam3CSK4 appears to be much stronger in fibroblasts compared to keratinocytes and suggest that TLR signaling pathways in fibroblasts may play important roles in the host defense against infection and/or in response to danger signals.

In conclusion, the present findings provide evidence for the constitutive expression levels of TLR family members and their functional responses to each TLR ligand in skin fibroblasts, and suggest that TLRs in skin fibroblasts may play an important role in the detection of and response to different classes of pathogens and/or danger signals.

\section{Acknowledgements}

The present study was supported by the National Research Foundation of Korea grant funded by the Korea Government (Ministry of Science, ICT and Future Planning) (no. 2009-0092835).

\section{References}

1. Akira S, Takeda $\mathrm{K}$ and Kaisho T: Toll-like receptors: critical proteins linking innate and acquired immunity. Nat Immunol 2: 675-680, 2001.

2. Medzhitov R: Toll-like receptors and innate immunity. Nat Rev Immunol 1: 135-145, 2001.

3. Takeda $\mathrm{K}$ and Akira S: Toll-like receptors in innate immunity. Int Immunol 17: 1-14, 2005.

4. Takeuchi O, Kaufmann A, Grote K, et al: Cutting edge: preferentially the R-stereoisomer of the mycoplasmal lipopeptide macrophage-activating lipopeptide-2 activates immune cells through a toll-like receptor 2- and MyD88-dependent signaling pathway. J Immunol 164: 554-557, 2000.

5. Takeuchi O, Sato $\mathrm{S}$, Horiuchi T, et al: Cutting edge: role of Toll-like receptor 1 in mediating immune response to microbial lipoproteins. J Immunol 169: 10-14, 2002.

6. Takeuchi O, Kawai T, Muhlradt PF, et al: Discrimination of bacterial lipoproteins by Toll-like receptor 6. Int Immunol 13: 933-940, 2001.

7. Alexopoulou L, Holt AC, Medzhitov R and Flavell RA: Recognition of double-stranded RNA and activation of NF-kappaB by Toll-like receptor 3. Nature 413: 732-738, 2001.

8. Tapping RI, Akashi S, Miyake K, Godowski PJ and Tobias PS: Toll-like receptor 4, but not toll-like receptor 2 , is a signaling receptor for Escherichia and Salmonella lipopolysaccharides. J Immunol 165: 5780-5787, 2000.

9. Hayashi F, Smith KD, Ozinsky A, et al: The innate immune response to bacterial flagellin is mediated by Toll-like receptor 5 . Nature 410: 1099-1103, 2001.

10. Heil F, Hemmi H, Hochrein $\mathrm{H}$, et al: Species-specific recognition of single-stranded RNA via toll-like receptor 7 and 8 . Science 303: 1526-1529, 2004.

11. Hemmi H, Takeuchi O, Kawai T, et al: A Toll-like receptor recognizes bacterial DNA. Nature 408: 740-745, 2000.

12. Sloane JA, Blitz D, Margolin Z and Vartanian T: A clear and present danger: endogenous ligands of Toll-like receptors. Neuromolecular Med 12: 149-163, 2010.

13. Calderwood SK, Mambula SS and Gray PJ Jr: Extracellular heat shock proteins in cell signaling and immunity. Ann NY Acad Sci 1113: 28-39, 2007. 
14. Bernard JJ, Cowing-Zitron C, Nakatsuji T, et al: Ultraviolet radiation damages self noncoding RNA and is detected by TLR3. Nat Med 18: 1286-1290, 2012.

15. Sasai $M$ and Yamamoto $M$ : Pathogen recognition receptors: ligands and signaling pathways by Toll-like receptors. Int Rev Immunol 32: 116-133, 2013.

16. Flacher V, Bouschbacher M, Verronese E, et al: Human Langerhans cells express a specific TLR profile and differentially respond to viruses and Gram-positive bacteria. J Immunol 177: 7959-7967, 2006.

17. Lebre MC, van der Aar AM, van Baarsen L, et al: Human keratinocytes express functional Toll-like receptor 3, 4, 5, and 9. J Invest Dermatol 127: 331-341, 2007.

18. Yu N, Zhang S, Zuo F, Kang K, Guan M and Xiang L: Cultured human melanocytes express functional toll-like receptors 2-4 7 and 9. J Dermatol Sci 56: 113-120, 2009.

19. Jang S, Park JS, Won YH, Yun SJ and Kim SJ: The expression of toll-like receptors (TLRs) in cultured human skin fibroblast is modulated by histamine. Chonnam Med J 48: 7-14, 2012.

20. Wang J, Hori K, Ding J, et al: Toll-like receptors expressed by dermal fibroblasts contribute to hypertrophic scarring. J Cell Physiol 226: 1265-1273, 2011.

21. Lee Y, Kim H, Kim S, Kim KH and Chung JH: Activation of toll-like receptors 2, 3 or 5 induces matrix metalloproteinase-1 and -9 expression with the involvement of MAPKs and NF-kappaB in human epidermal keratinocytes. Exp Dermatol 19: e44-e49, 2010.

22. McMillan SJ, Kearley J, Campbell JD, et al: Matrix metalloproteinase- 9 deficiency results in enhanced allergen-induced airway inflammation. J Immunol 172: 2586-2594, 2004

23. Lee YM, Li WH, Kim YK, Kim KH and Chung JH: Heat-induced MMP-1 expression is mediated by TRPV1 through PKCalpha signaling in HaCaT cells. Exp Dermatol 17: 864-870, 2008.

24. Kollisch G, Kalali BN, Voelcker V, et al: Various members of the Toll-like receptor family contribute to the innate immune response of human epidermal keratinocytes. Immunology 114: 531-541, 2005.

25. Rittié L and Fisher GJ: UV-light-induced signal cascades and skin aging. Ageing Res Rev 1: 705-720, 2002.
26. Fisher GJ, Wang ZQ, Datta SC, Varani J, Kang S and Voorhees JJ: Pathophysiology of premature skin aging induced by ultraviolet light. N Engl J Med 337: 1419-1428, 1997.

27. Wang XY, Tao CJ, Wu QY and Yuan CD: Protein extract of ultraviolet-irradiated human skin keratinocytes promote the expression of mitogen-activated protein kinases, nuclear factor-kappaB and interferon regulatory factor-3 in Langerhans cells via Toll-like receptor 2 and 4 . Photodermatol Photoimmunol Photomed 29: 41-48, 2013.

28. Vabulas RM, Ahmad-Nejad P, da Costa C, et al: Endocytosed HSP60s use toll-like receptor 2 (TLR2) and TLR4 to activate the toll/interleukin-1 receptor signaling pathway in innate immune cells. J Biol Chem 276: 31332-31339, 2001.

29. Libermann TA and Baltimore D: Activation of interleukin-6 gene expression through the NF-kappa B transcription factor. Mol Cell Biol 10: 2327-2334, 1990.

30. Elliott CL, Allport VC, Loudon JA, Wu GD and Bennett PR: Nuclear factor-kappa B is essential for up-regulation of interleukin-8 expression in human amnion and cervical epithelial cells. Mol Hum Reprod 7: 787-790, 2001.

31. Siebenlist U, Franzoso G and Brown K: Structure, regulation and function of NF-kappa B. Annu Rev Cell Biol 10: 405-455, 1994.

32. Liu-Bryan R, Scott P, Sydlaske A, Rose DM and Terkeltaub R: Innate immunity conferred by Toll-like receptors 2 and 4 and myeloid differentiation factor 88 expression is pivotal to monosodium urate monohydrate crystal-induced inflammation. Arthritis Rheum 52: 2936-2946, 2005.

33. Bhattacharyya S, Kelley K, Melichian DS, et al: Toll-like receptor 4 signaling augments transforming growth factor-beta responses: a novel mechanism for maintaining and amplifying fibrosis in scleroderma. Am J Pathol 182: 192-205, 2013.

34. Niebuhr M, Baumert K and Werfel T: TLR-2-mediated cytokine and chemokine secretion in human keratinocytes. Exp Dermatol 19: 873-877, 2010.

35. Meisgen F, Xu Landen N, Wang A, et al: MiR-146a negatively regulates TLR2-induced inflammatory responses in keratinocytes. J Invest Dermatol 134: 1931-1940, 2014. 